\title{
Feedback-Error Learning for Gait Rehabilitation Using a Powered Knee Orthosis: First Advances*
}

\author{
Pedro Nuno Fernandes, Joana Figueiredo, Juan C. Moreno and Cristina P. Santos
}

\begin{abstract}
Powered assistive devices have been playing a major role in gait rehabilitation. Hereby, the development of time-effective control strategies to manage such devices is a key concern to rehabilitation engineering. This paper presents a real-time Feedback-Error Learning control strategy, by means of an Artificial Neural Network as a feedforward controller to acquire the inverse model of the plant, and a ProportionalIntegral-Derivative feedback controller to guarantee stability and handle with disturbances. A Powered Knee Orthosis was used as the assistive device and a trajectory generator assistive strategy, previously acquired through an inertial system, was applied. A validation with one subject walking in a treadmill at $1 \mathrm{~km} / \mathrm{h}$ with the Powered Knee Orthosis controlled by the Feedback-Error Learning control was performed. Evidences on the control behavior presented good performances, with the Artificial Neural Network taking 90 seconds to learn the inverse model, which enabled a decrease in the angular position error by $75 \%$ and eliminated the phase delay, when compared to solo Proportional-Integral-Derivative feedback controller. Robust reactions to external disturbances were also achieved. The implemented Feedback-Error Learning strategy proves to be a time-effective asset to control assistive powered devices.
\end{abstract}

\section{INTRODUCTION}

Traditional gait rehabilitation is progressively being complemented with powered assistive devices to treat physical limb impairments [1], [2]. Particularly, powered lower-limb exoskeletons and orthoses have been highlighted, as they increase human motor function, build up joint strength and rehabilitate through task-oriented training, acting in parallel with the user lower limb [3], [4].

Furthermore, the human motor control system starts to influence the design of bioinspired architectures for lowerlimb robotic devices, with emphasis to the human-machine interface [5]. Assistive strategies that ensure repetitive movements of the user's limbs to, improve the movement

*This work has been supported in part by the Fundação para a Ciência e Tecnologia (FCT) with the Reference Scholarship under Grant SFRH/BD/108309/2015, and part by the FEDER Funds through the Programa Operacional Regional do Norte and national funds from FCT with the project SmartOs -Controlo Inteligente de um Sistema Ortótico Ativo e Autónomo- under Grant NORTE-01-0145-FEDER-030386, and by the FEDER Funds through the COMPETE 2020 - Programa Operacional Competitividade e Internacionalização (POCI) - with the Reference Project under Grant POCI-01-0145-FEDER-006941 and supported by grant RYC2014-16613 by Spanish Ministry of Economy and Competitiveness.

Pedro Nuno Fernandes, Joana Figueiredo, and Cristina P. Santos are with Center for MicroElectroMechanical Systems (CMEMS), University of Minho, Guimarães, Portugal (e-mail: \{a68385,id6003\}@alunos.uminho.pt, cristina@dei.uminho.pt).

Juan C. Moreno is with Neural Rehabilitation Group, Cajal Institute, Spanish National Research Council, Madrid, Spain (e-mail: jc.moreno@csic.es). coordination and control strategies capable of providing adequate responses should be a concern. For this matter, tracking control strategies can be developed to integrate mechanisms based on predefined trajectories [6]. These assistive strategies aim to predefine the joint trajectory through the gait analysis of limbs movement and/or joints kinematics [7]. Feedback control laws, such as the Proportional-Derivative (PD) and Proportional-IntegralDerivative (PID), are commonly implemented to perform the set trajectory tracking due to its feasibility and easy to implement [1], [8], [9]. Nevertheless, measurement noise in the feedback loop, actuation saturation in fast changing systems and changes in the process dynamics are limitations that affect these control laws and can lead the system to instability [10].

In order to overcome these drawbacks, a control law with a feedforward block responsible to deal with the response to trajectory tracking and a feedback block to handle with disturbances and uncertainties gives a good solution to the control problem. For instance, Kawato [11] suggested the Feedback-Error Learning (FEL) control law. It uses an Artificial Neural Network (ANN) as feedforward block to learn the inverse dynamic model of the controlled plant, by means of the performed feedback command output. Kawato ground his study on the human central nervous system, describing the FEL law as an inverse model learning method for the cerebellum [11].

The goal of the current work was to design, tune and validate a real-time FEL control, using ANN as feedforward controller to acquire the inverse robotic model. A tuned PID was used to provide the feedback motor command, to guarantee stability during the real-time learning process, and to handle with disturbances after the training procedure is completed. The FEL control law was tuned and validated in a powered assistive device [12], using a trajectory tracking assistive strategy for gait rehabilitation of the knee joint. The applied control was designed using normalized inputs to handle with variations in the generated trajectory, allowing the application of this control in several user-oriented trajectories.

\section{METHODS}

The implemented real-time FEL control was developed in the STM32F4 Discovery Board, as well as the trajectory tracking assistive strategy that generates the knee trajectory. This control was validated with a healthy subject, walking in a treadmill. Fig. 1 illustrates de developed strategy.

\section{A. Powered Knee Orthosis}

The assistive device is a powered right knee orthosis, a module of the lower limb robotic H2-exoskeleton (Technaid 
S.L., Spain), a full-body system designed to assist stroke survivors [6]. It has embedded an electronic actuator (DC brushless motor) and sensors (potentiometer, among others). For more details about the hardware and software of the device see Félix et al. [12].

\section{B. Trajectory Tracking Assistive Strategy}

According to [5], the high-level control of bioinspired control architectures for lower-limb robotic devices, implements the perception layer, where the user's state is inferred to adjust the motion assistance. Therefore, an inertial system was used to acquire the user's right knee trajectory, so the trajectory generator could use it as reference. As seen in Fig. 1, according to the desired velocity and target joint, the trajectory generator system in the high-level yields the reference angular positions. With this kind of assistive strategy, repetitive motions are established during the rehabilitation therapy, improving muscular strength and movement coordination in neurological patients [6].

\section{Feedback-Error Learning}

At the lower level of the bioinspired control architecture, the desired states provided by the high-level layer are tracked. The FEL control is responsible to infer about the correct control commands, in order to perform the intended movement by means of a tuned PID feedback controller and a feedforward controller comprised by an ANN with the trained plant's inverse model.

\section{1) Feedback controller}

The discrete PID feedback controller implements the control law presented in (1), where $e_{k}$ and $e_{k-1}$ corresponds to the current and previous error between the reference and measured angular position. The Ziegler-Nichols method was applied to find the correct controller gains $\left(K_{p}, K_{i}\right.$, and $\left.K_{d}\right)$.

$$
u_{f b}=K_{p} e_{k}+K_{i} \sum_{n=1}^{k} e_{n} \Delta t+K_{d} \frac{e_{k}-e_{k-1}}{\Delta t}
$$

The feedback controller is responsible to provide the

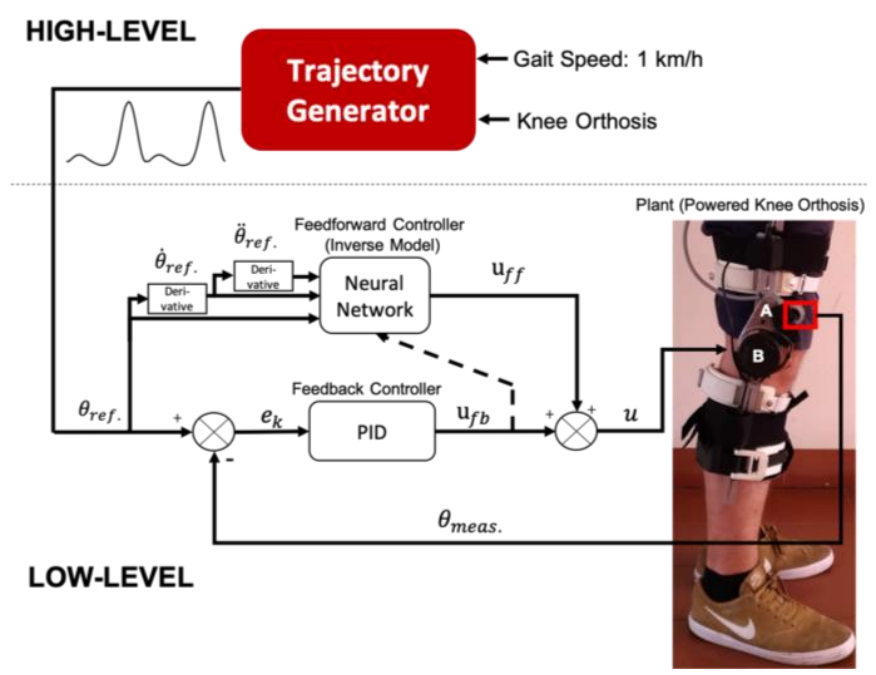

Figure 1 - Implemented FEL strategy for trajectory gait rehabilitation, where $\theta_{\text {meas. }}$ is the measured angular position $\theta_{\text {ref. }}$ is the reference angular position, $\dot{\theta}_{\text {ref }}$. is the reference angular velocity, $\ddot{\theta}_{\text {ref. }}$ is the reference angular acceleration, $e$ is the position error, $u_{f b}$ is the feedback command, $u_{f f}$ is the feedforward command, $u$ the total control command, $\mathrm{A}$ is the potentiometer and $\mathrm{B}$ the actuator. feedback commands to the feedforward controller and to manage with disturbances that could affect the system.

\section{2) Feedforward controller}

The feedforward controller aims to gather the inverse model of the plant. The chosen method to infer it, was the implementation of an ANN due to its proper estimation performance, good generalization and capabilities to map non-linearities [13]. Multi-Layer Perceptron (MLP) was the adopted structure for the network, where each neuron is only connected to every neuron in the adjacent layer and the supervised learning was the chosen training method. The approach taken for the weights update was the backpropagation algorithm, that is based on the minimization of the gradient descent of the error's prediction with respect to the network's weights change. To implement it, the stochastic gradient descent (SGD) approach was used, where the weights are updated on each online training iteration. Table 1, identifies the chosen set-up for the ANN.

TABLE I. ANN'S CHARACTERISTICS.

\begin{tabular}{|c|c|c|c|c|}
\hline $\begin{array}{c}\text { Input } \\
\text { Nodes }\end{array}$ & $\begin{array}{c}\text { Hidden } \\
\text { Nodes }\end{array}$ & $\begin{array}{c}\text { Output } \\
\text { Nodes }\end{array}$ & Training Method & Learning rate \\
\hline 3 & 4 & 1 & $\begin{array}{c}\text { Supervised, } \\
\text { Backpropagation } \\
\text { SGD }\end{array}$ & $\begin{array}{c}\text { Adaptive }(0.0001 \\
\text { and lower })\end{array}$ \\
\hline
\end{tabular}

The supplied input values for the ANN were the reference angular position $\left(\theta_{\text {ref. }}\right)$, which is derived to obtain velocity $\left(\dot{\theta}_{\text {ref. }}\right)$ and acceleration $\left(\ddot{\theta}_{\text {ref. }}\right)$. A normalization method to scale the data between $[-1 ; 1]$ was performed, as it reduces the estimation error, accelerates the training phase [14]. Moreover, it provides versatility to the training and testing by handling with variations in the generated trajectory and speed, allowing the application of this control in several user-oriented trajectories.

The provided feedback command $\left(u_{f b}\right)$ acts as the error values for the ANN to decrease in order to acquire the plant's inverse model and provide feedforward commands $\left(u_{f f}\right)$.

The implemented bioinspired real-time control observed in Fig. 1 runs at $1 \mathrm{kHz}$, making it a time-effective strategy. Each reference value is updated at every $72 \mathrm{~ms}$, establishing a gait cycle with $3.5 \mathrm{~s}$, imposing the desired $1 \mathrm{~km} / \mathrm{h}$ gait velocity.

\section{Validation}

Firstly, a validation without a user (with the PKO deprived of load) was performed to investigate the behavior of the FEL control.

In the target validation, a user was asked to walk at 1.0 $\mathrm{km} / \mathrm{h}$ in a level-ground on a treadmill, with the PKO configured with the trajectory tracking assistive strategy. In the last phase of the experiment and under the same conditions as before, the user was asked to counter the PKO movement in the terminal stance phase (before toe-off event) in order to evaluate how the controller would react to such disturbance, which may occur during a gait therapy.

\section{RESUlTS \& DISCUSSION}

In order to validate the FEL control, we analyze the following signals: (1) PKO trajectory measured by a 

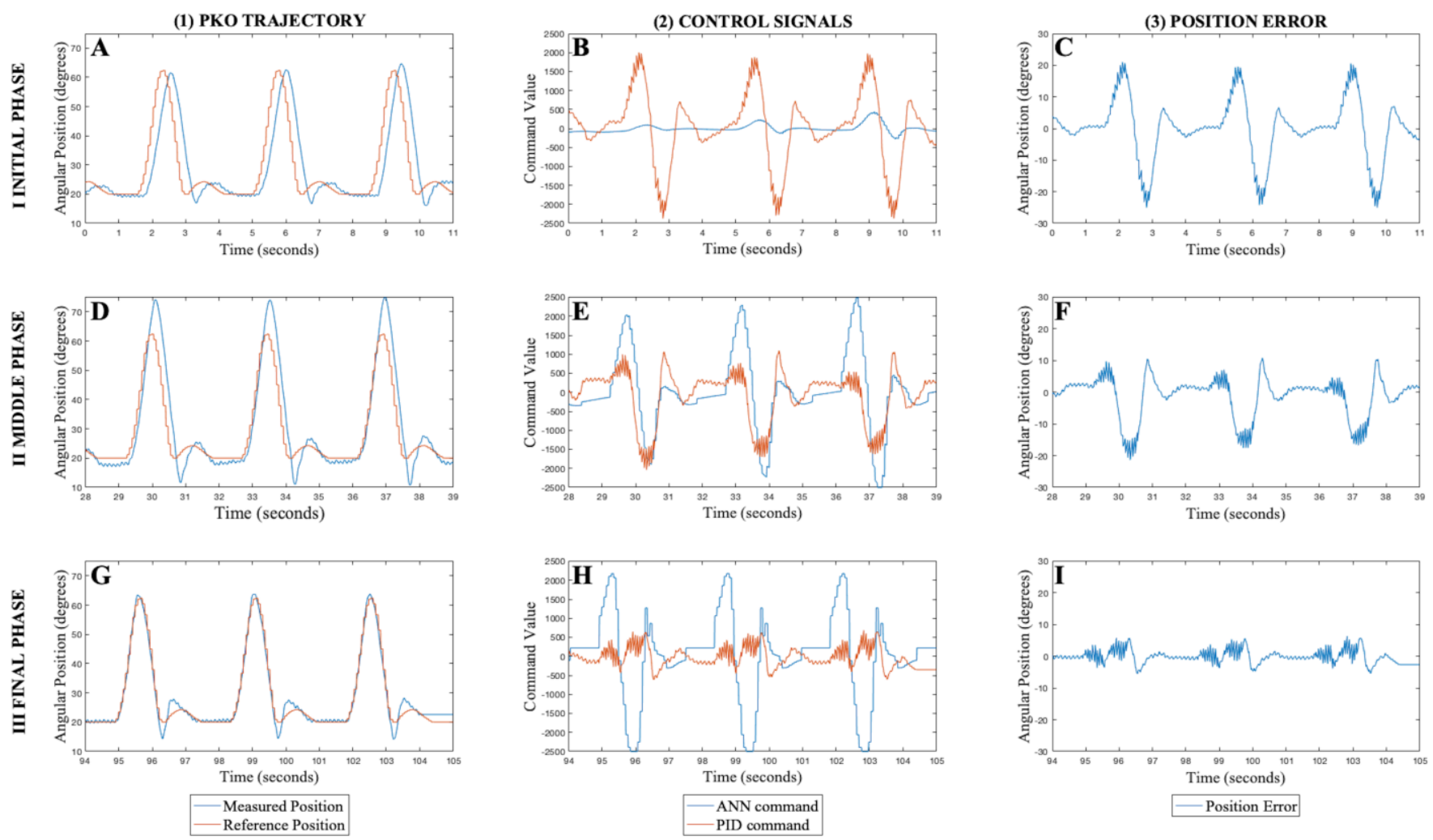

Figure 2 - FEL performance control signals. A, D, G: PKO Measured trajectory and Reference trajectory signals in the Initial, Middle, and Final Phase, respectively; B, E, H: ANN and PID command signals in the Initial, Middle, and Final Phases, respectively; C, F, I: PKO Position Error in the Initial, Middle, and Final Phase.

potentiometer and reference trajectory; (2) PID and ANN command and (3) Evaluation of PKO position error. All signals were sampled with $100 \mathrm{~Hz}$ of frequency. Fig. 2 depicts the acquired signals under three different gait cycle periods: I Initial Phase; II Middle Phase and III Final Phase.

The first row displays the trajectory, control and error signals in the Initial Phase (first $11 \mathrm{~s}$ ) of the FEL control. During this phase, the feedforward controller is starting to tune its ANN and its contribution to the total control signal $(u)$ it is not significant, being the feedback PID controller the responsible to track the reference trajectory. In the second row, the FEL control has performed several iterations, having passed about $40 \mathrm{~s}$ (Middle Phase). The feedforward has much more relevance to the overall control command, although it is in the middle phase of tuning process. Lastly, in the lower row (Final Phase) of signals in Fig. 2, the FEL control has learned the plant's inverse model dynamic, and it is the main contributor to the total control signal.

Regarding the Initial Phase, it is possible to see in Fig. 2.A that, the measured trajectory has approximately $0.21 \mathrm{~s}$ of delay to the generated reference signal. This outcome is produced mainly by the PID control, once the feedforward controller is in the initial learning phase, having a much lower influence in the total control signal (Fig 2.B). Hence, the error signal in Fig. 2.C has a variance of roughly $20^{\circ}$.

As the FEL controller initiates to learn the inverse model of the PKO, the measured position starts to decrease the phase difference with the generated reference signal, as can be seen in Fig. 2.D. However, in order to correct its phase, the controller produces signals that causes the PKO to perform a trajectory with $10^{\circ}$ more of amplitude, as stated in Fig. 2.D. This is due to the fact that the ANN has not leaned the inverse model with the best performance yet, being in the middle phase of its training. In Fig. 2.E, it is possible to realize the increase in the ANN command signal, and that, respectively, the PID command signal has decreased in relation to its previous state. The error signal given in Fig. 2.F has now improved, varying from 10 to -20 degrees of angular position.

By observing Fig.2.G, we verified that the FEL control has successfully aligned the PKO trajectory with the reference trajectory and corrected the amplitude divergence. This state was observed about $90 \mathrm{~s}$ from the start of the FEL control strategy. In this phase, the dynamic inverse model of the plant has been adequately obtained by the ANN, being the feedforward controller the main grantor of the total control command - Fig.2.H. The PID command has now a mean feedback command signal of $4.4 \%$ in regard to the feedback command, meaning that has decrease $95.6 \%$ concerning its initial phase. Respecting the error value, this signal has decreased about 75\%, comparing Fig. 2.C and Fig. 2.I.

Table II reviews the discussed results, analyzing the Root Mean Squared Error (RMSE) and phase delay between the reference and measured trajectories, and the contributions of the feedforward control $\left(u_{f f}\right)$ and feedback control $\left(u_{f b}\right)$, in the Initial, Middle and Final Phase of the FEL controller. 

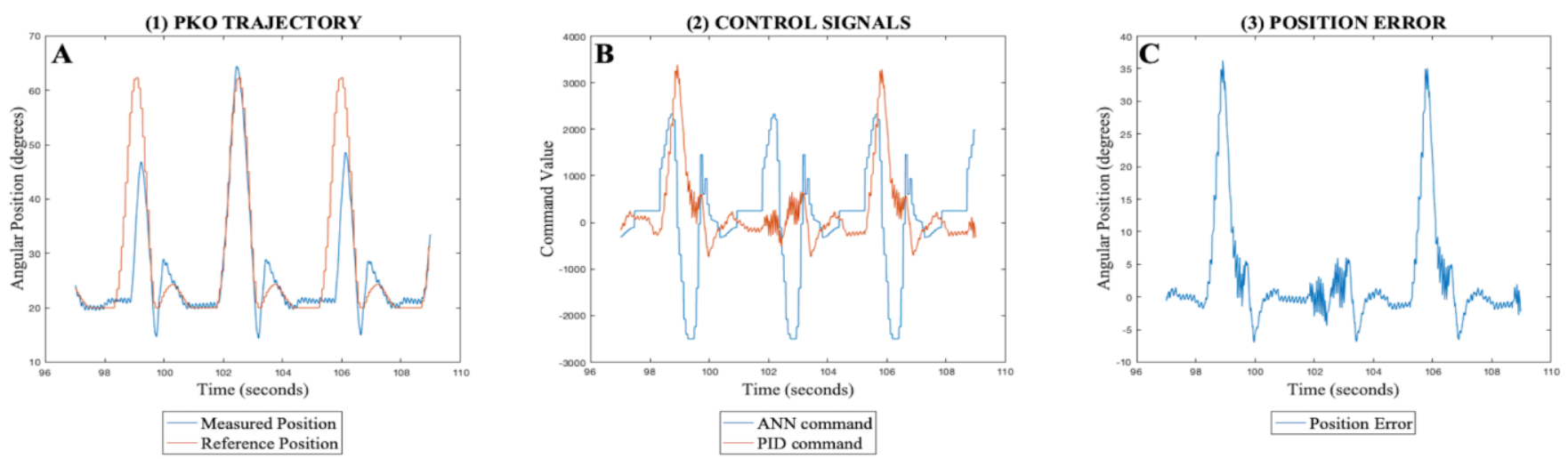

Figure 3 - FEL performance to disturbances. A: PKO Measured and Reference position signals; B: ANN and PID command signals; C: PKO Position Error; All signals were acquired with the FEL at final phase, i.e., with the inverse model of the plant inferred.

TABLE II. FEL CONTROL Performance.

\begin{tabular}{|c|c|c|c|c|}
\hline $\begin{array}{c}\text { FEL } \\
\text { Phase }\end{array}$ & $\begin{array}{c}\text { Position } \\
\text { RMSE }(\%)\end{array}$ & $\begin{array}{c}\text { Delay } \\
(\boldsymbol{s})\end{array}$ & $\begin{array}{c}\boldsymbol{u}_{f f} \text { Contribution } \\
(\%)\end{array}$ & $\begin{array}{c}\boldsymbol{u}_{f b} \text { Contribution } \\
(\%)\end{array}$ \\
\hline Initial & 18 & -0.21 & 0 & 100 \\
\hline Middle & 19 & -0.06 & 60 & 40 \\
\hline Final & 10 & 0 & 95.6 & 4.4 \\
\hline
\end{tabular}

\section{A. Performance to Disturbances}

Fig. 3 shows the performance of the FEL control to the disturbances applied to the PKO, after the acquisition of the inverse model. The analyzed signals were the same as before, i.e., the: (1) PKO measured and reference trajectory; (2) PID and ANN command and (3) Evaluation of PKO position error.

With the learned inverse model, it was possible to analyze the behavior of the FEL controller to disturbances that could affect its performance. Hereby, in Fig. 3.A it is possible to inspect the moments when the user countered the orthosis movement, preventing it from increasing its angular position, in two different occasions (98 s and $205 \mathrm{~s}$ ). Looking to Fig. 3.B, in that same moments, the PID command $\left(u_{f b}\right)$ signal increases, once the error signal grows (see Fig. 3D) due to the displacement between the reference and measured position signals. Respecting the command signal performed by the feedforward controller, it stayed periodic, as the reference signals and the learned inverse model did not change.

Therefore, the feedback controller successfully canceled the increased error signal and corrected the PKO angular position, preventing the FEL control from fall into an instability state and from damaging the user and the device.

\section{CONCLUSIONS \& FUtURE WORK}

This paper implements a real-time FEL control, using an ANN and PID to control a PKO. The outcomes highlight the improvement of the studied strategy over the solo implementation of a PID feedback controller. It is verified a substantial decrease in the position error signal with the elimination of the phase delay, with low training time to acquire the inverse model of the system. Moreover, the PID feedback handles with random disturbances in normal gait and the $\mathrm{NN}$ is capable of adjusting to the user-oriented trajectory when needed. These findings support that the integration of FEL control in the low-level architecture of assistive devices may yield more time-effective assistive strategies and consequently, well-performed gait training.

Future work comprises the validation of the stated strategy with more subjects and different walking conditions, such as walking speeds and slopes. Also, its analysis with distinct high-level controllers and powered medical devices will be addressed.

\section{REFERENCES}

[1] S. Viteckova, P. Kutilek, and M. Jirina, "Wearable lower limb robotics: A review.," Biocybern. Biomed. Eng., vol. 33, no. 2, pp. 96-105, 2013.

[2] K. Anam and A. A. Al-jumaily, "Active Exoskeleton Control Systems : State of the Art," Int. Symp. Robot. Intell. Sensors, vol. 41, pp. 988-994, 2012.

[3] H. Herr, "Exoskeletons and orthoses: classification, design challenges and future directions.," J. Neuroeng. Rehabil., vol. 6, p. 21, 2009.

[4] A. M. Dollar and H. Herr, "Lower Extremity Exoskeletons and Active Orthoses: Challenges and State-of-the-Art.," IEEE Trans. Robot., vol. 24, no. 1, pp. 144-158, 2008.

[5] M. R. Tucker, "Control strategies for active lower extremity prosthetics and orthotics: a review," J. Neuroeng. Rehabil., vol. 12, no. 1, p. 1, 2015.

[6] M. Bortole et al., "The $\mathrm{H} 2$ robotic exoskeleton for gait rehabilitation after stroke: early findings from a clinical study," $J$. Neuroeng. Rehabil., vol. 12, no. 1, p. 54, 2015.

[7] J. Cao, S. Q. Xie, R. Das, and G. L. Zhu, "Control strategies for effective robot assisted gait rehabilitation: The state of art and future prospects," Med. Eng. Phys, vol. 36, no. 12, pp. 15551566, 2014

[8] T. Yan, M. Cempini, C. M. Oddo, and N. Vitiello, "Review of assistive strategies in powered lower-limb orthoses and exoskeletons," Rob. Auton. Syst., vol. 64, pp. 120-136, 2015.

[9] K. Anam and A. A. Al-jumaily, "Active Exoskeleton Control Systems : State of the Art," Int. Symp. Robot. Intell. Sensors, pp 988-994, 2012.

[10] W. Lu, J. Yang, and X. Liu, "The PID controller based on the artificial neural network and the differential evolution algorithm," J. Comput., vol. 7, no. 10 SPL.ISS., pp. 2368-2375, 2012.

[11] M. Kawato, "Feedback-error-learning neural network for supervised motor learning," Adv. neural Comput., vol. 6, no. 3, pp. 365-372, 1990.

[12] P. Felix, J. Figueiredo, C. P. Santos, and J. C. Moreno, "Eletronic Design and Validation of Powered Knee Orthosis System with Werable Sensors," 17th Int. Conf. Auton. Robot Syst. Compet. (ICARSC 2017), 2017.

[13] A. P. E. Meireles Magali RG, "A comprehensive review for industrial applicability of ANNs.," IEEE Trans Ind Electron, vol. 50 , no. 3, pp. 585-601, 2003

[14] J. Sola and J. Sevilla, "Importance of input data normalization for the application of neural networks to complex industrial 
problems," IEEE Trans. Nucl. Sci., vol. 44, no. 3 PART 3, pp. 1464-1468, 1997. 